\title{
Commemoration of Lidia Barbanera
}

\section{Franco Giovannelli ${ }^{* \dagger}$}

INAF - Istituto di Astrofisica e Planetologia Spaziali, Via del Fosso del Cavaliere, 100, 00133

Roma, Italy

E-mail: franco.giovannellidiaps.inaf.it

In this short paper I celebrate the life of Lidia Barbanera. Lidia was among the historical colleagues from my own old institute (Laboratorio di Astrofisica Spaziale, CNR). We started to collaborate directly beginning in 1984 when I organized the first historical Frascati Workshop on "Multifrequency Behaviour of Galactic Accreting Sources". Lidia was the workshop secretary, and the factotum during the preparation and administration of the event. Only Lidia and I were involved in the organization of such "new multifrequency workshops".

This brief remembrance does not list the many important contributions of Lidia within the many activities of my institute, but simply paints his humanity through a few episodes of daily life that will stay in my mind forever.

Frontier Research in Astrophysics - II

23-28 May 2016

Mondello (Palermo), Italy

* Speaker.

${ }^{\dagger}$ A footnote may follow. 


\section{In memoriam}

Lidia was a good technical/administrative collaborator, but above all a peaceful, kind and courteous woman, although sometimes very nervous, perhaps almost rude, when something was going wrong because of lack of manners of interlocutor, or even worse when the interlocutor was arrogant.

One particularly good memory stand out when she went with me once to Madrid on the occasion of one of my many IUE shifts. I introduced Lidia to my good friends Jaime and Angela, both professional painters. Figure 1 shows the "Fiesta" after a dinner in Calle Toledo. Left panel shows Jaime, Angela and myself; central panel shows Lidia with a group of carefree friends at dinner: Lidia is at the end of the table in the center; right panel shows Lupe dancing a "sevillana", Angela clapping her hands, accompanying the cheerful music of Jaime's guitar.
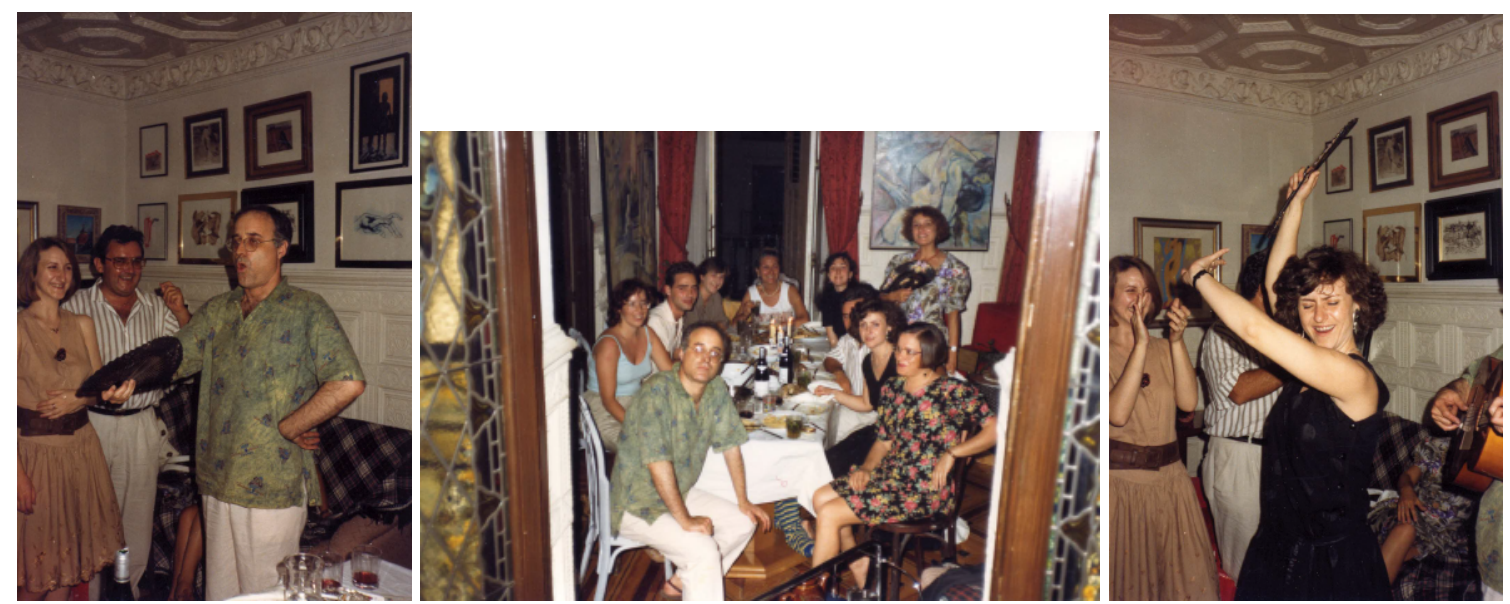

Figure 1: Left panel: Jaime, Angela and Franco; central panel: dinner table with a group of carefree friends - Lidia is at the end of the table in the center; right panel: Lupe dancing a "sevillana", Angela clapping her hands, accompanying the cheerful music of Jaime's guitar

Lidia was also the managing secretariat of the Vulcano Workshops series on "Frontier Research in Astrophysics and Particle Physics", and was always sharing with us all the pleasures of the table, as shown in Figure 2 (left panel) where Lidia, smiling, is to the right in foreground. She was also happy to share with her friends Franco and Lola - the editors of the Frascati Workshops proceedings - some happy moments, like shown in the Figure 2 (right panel).

Lidia also served as managing secretariat of the two workshops on "The Bridge between the Big Bang and Biology", the first in Stromboli Island (1999) and the second in Vulcano Island (2002) (Aeolian Archipelago). These workshops were a clear example of a multidisciplinary approach for studying the evolution of the Universe from the Big Bang to the emergence of life.

I remember with particular fondness the happy moments during the Millimetron Workshop 2010 "Science with Millimetron" held in Palermo from 14th to 18th June. The venue for the workshop was the Splendid Hotel La Torre in Mondello where you can admire the beautiful panorama of Mount Pellegrino in the background (Figure 3, left panel) and Lidia gently holding a sunflower (Figure 3, right panel). 

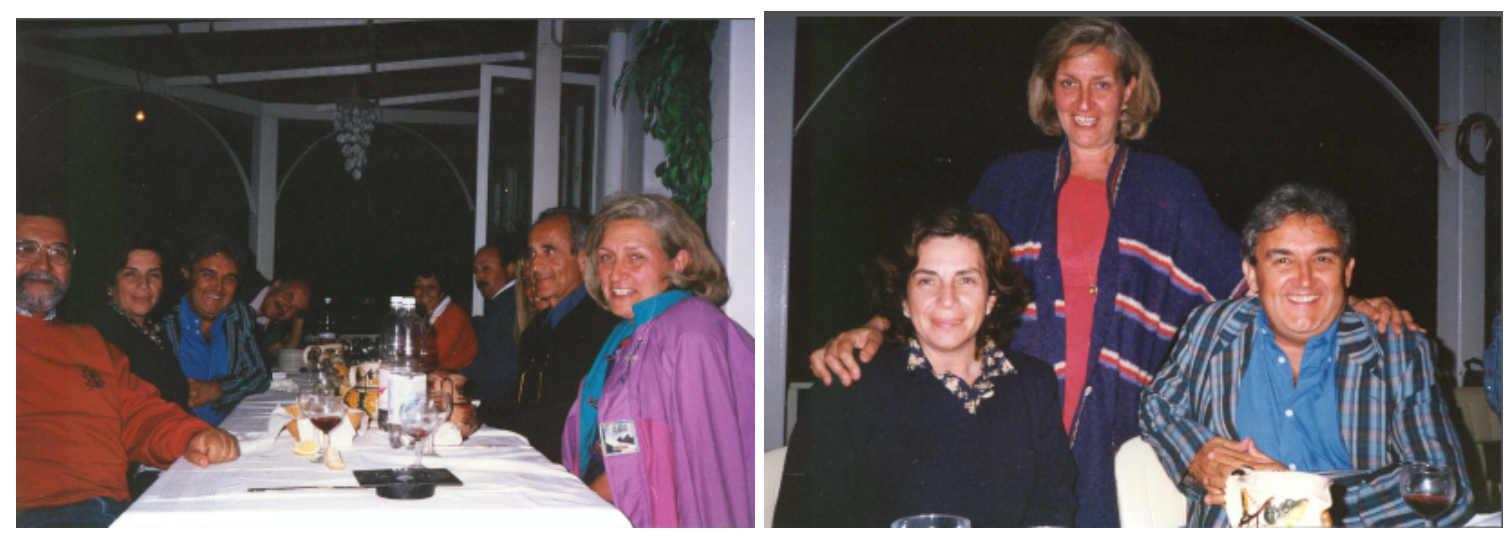

Figure 2: Left panel: Lidia Barbanera smiling in foreground on the right. Right panel: Lidia smiling in between Lola and Franco.
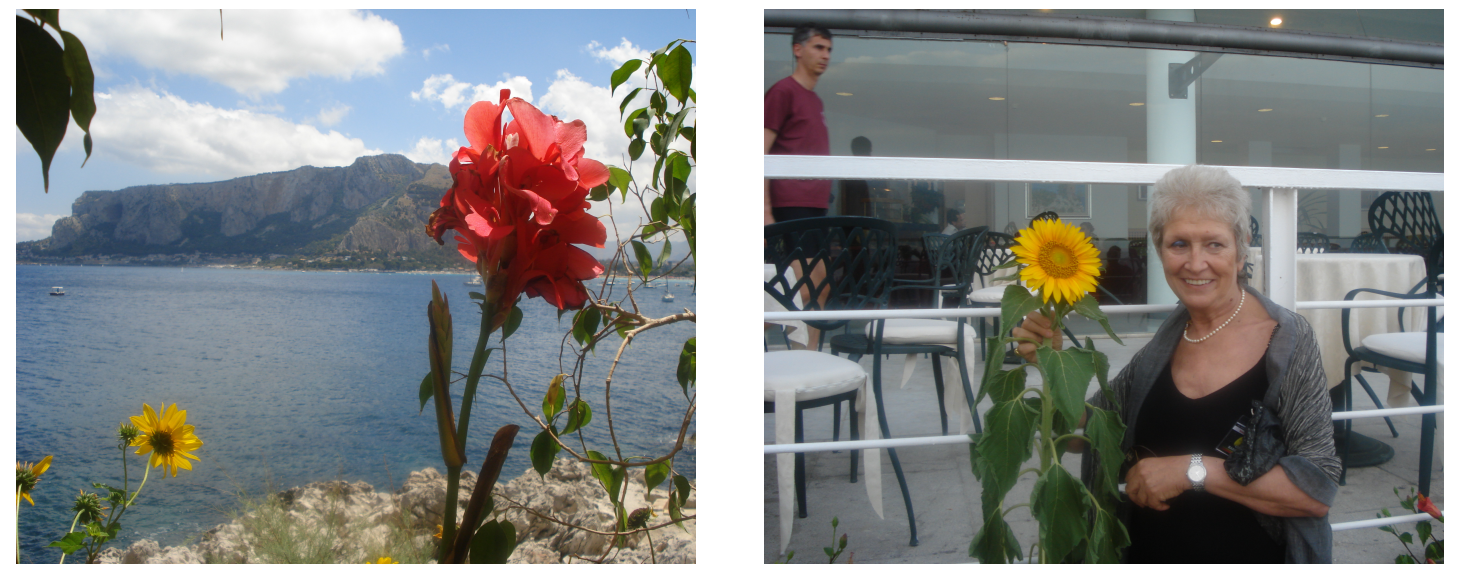

Figure 3: Left panel: Panorama with Mount Pellegrino. Right panel: Lidia softly holding a sunflower.

After the concert for piano and violin of Maestro Alessandro Perpich (violin) and Maestra Valentina Usai (piano), Lidia, smiling, offered a tribute to Maestro Alessandro Perpich (Figure 4,
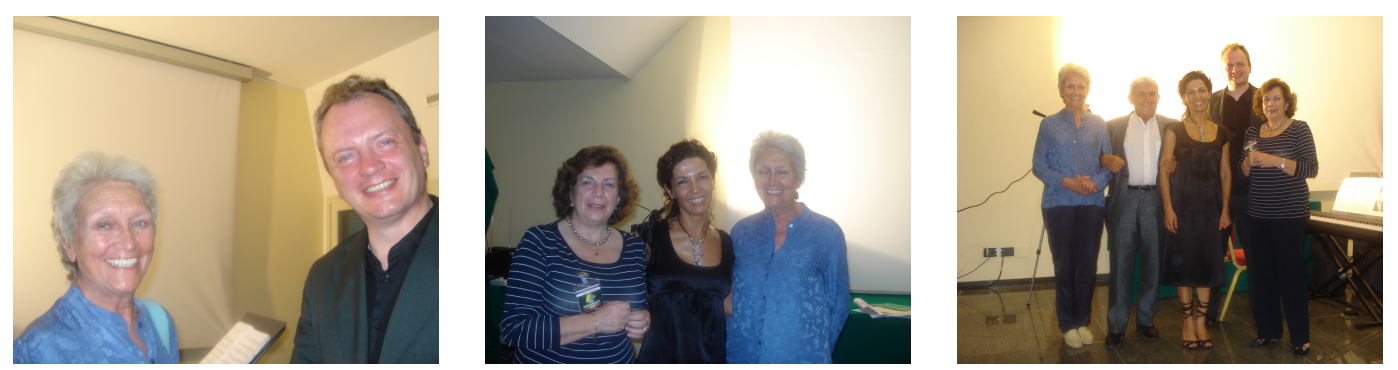

Figure 4: Left panel: Lidia honours Maestro Alessandro Perpich. Central panel: Lidia, Maestra Valentina Usai, and Lola. Right panel: Lidia with Maestri Alessandro Perpich and Valentina Usai, and the organizers Franco and Lola. 
left panel). Lidia, Lola and Valentina after the concert (Figure 4, central panel), and finally Lidia with Maestri Valentina and Alessandro, and Franco and Lola, the organizers (Figure 4, right panel).

Lidia felt completely at ease throughout the week, as shown in the various panels of Figures 5.
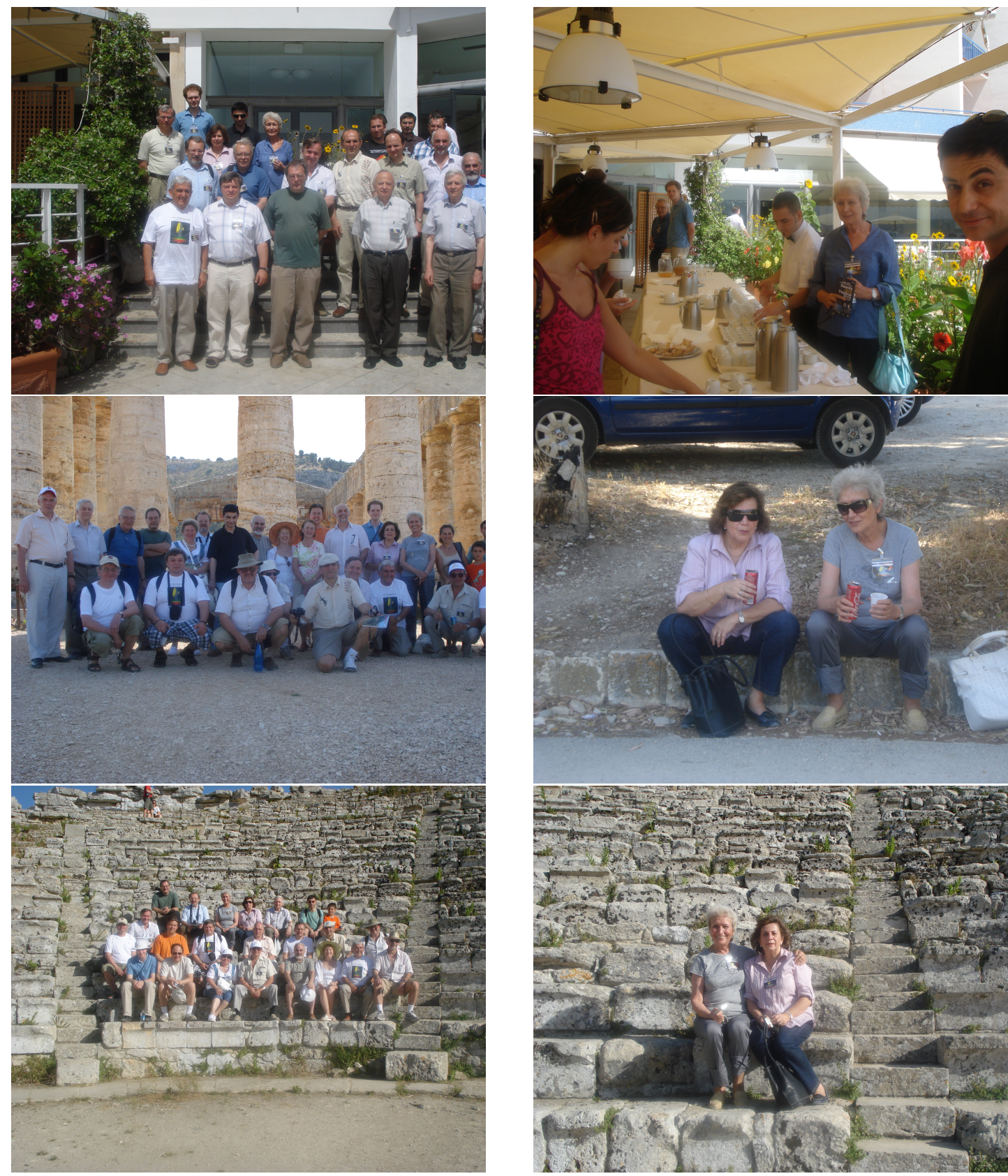

Figure 5: Left upper panel: Participants, with Lidia in the center of the last line. Right upper panel: Lidia during a coffe break. Left central panel: Participants in Segesta at the Greek Temple - Lidia with blue dress. Right central panel: Lola and Lidia resting in Segesta. Left lower panel: Participants in the Greek Theatre in the last line Lidia in between Lola and Memmo Federici, our good colleague and friend passed away on 5th July 2016. Right lower panel: Lidia and Lola in the Greek Theatre. 
Lidia was managing the secretariat of our Frascati workshops ("Multifrequency Behaviour of High Energy Cosmic Sources") till 2011 when she started to fight against her awful illness. Figure 6 shows Lidia, smiling, at the Vulcano registration desk at the hotel Eolian, together with Nino Panagia, a veteran participant in our workshops, as well as an old member of the Frascati Astrophysical Laboratory.

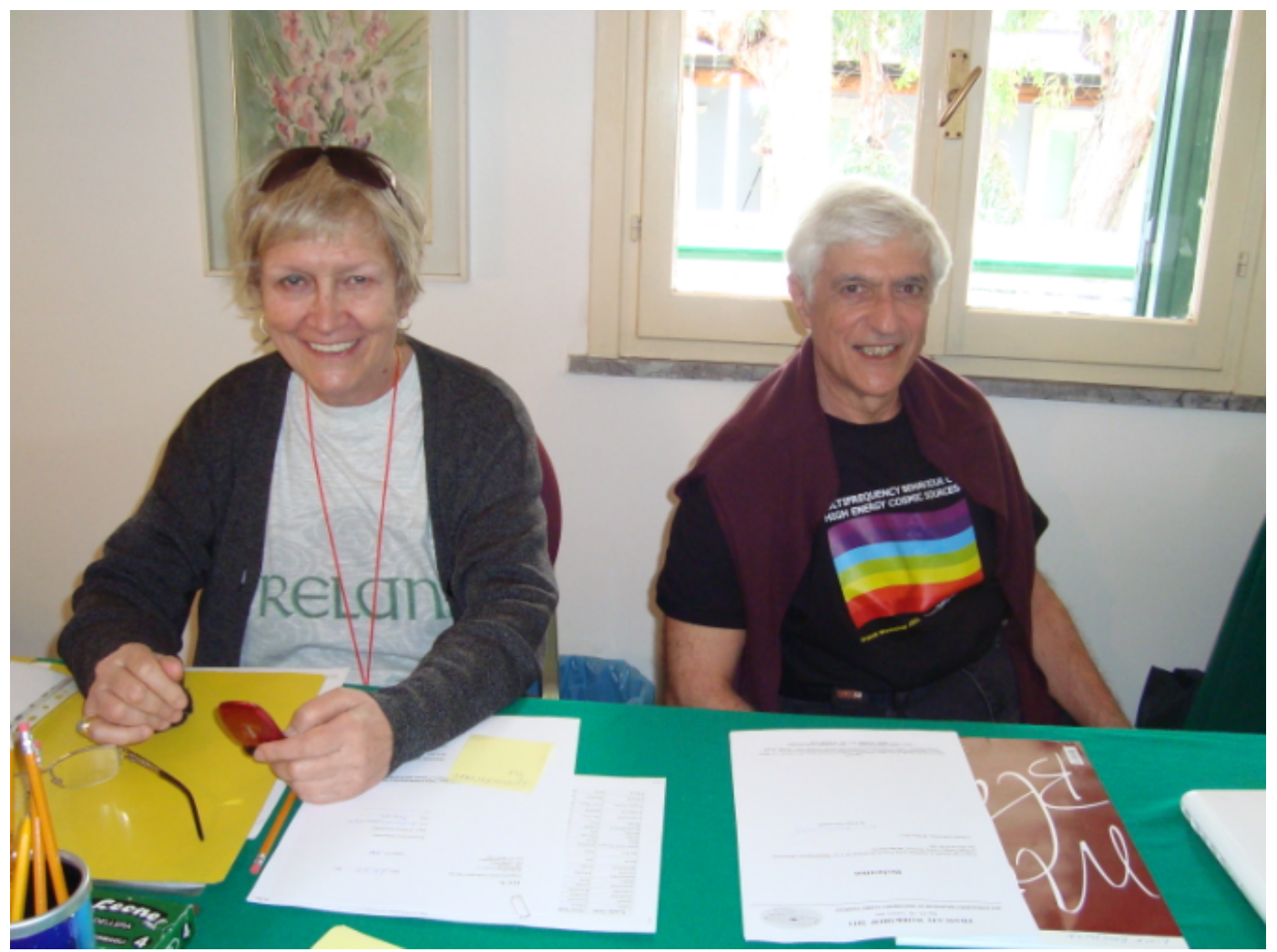

Figure 6: Lidia Barbanera (Roma, 7th April 1942 - Roma, 6th October 2015). Her last image in Vulcano with Nino Panagia.

This Frontier Research in Astrophysics workshop is the first one I organized without Lidia's presence in life. I'm really sorry for her absence.

Dear Lidia, on behalf of all your friends, may you rest in peace. 